А. А. Машанов, В. В. Мантатов, Д. С. Сандитов. О температурной зависимости энергии активации процесса стеклования

УДК 541.64:536.4

DOI 10.18101/2306-2363-2019-4-47-54

\title{
О ТЕМПЕРАТУРНОЙ ЗАВИСИМОСТИ ЭНЕРГИИ АКТИВАЦИИ ПРОЦЕССА СТЕКЛОВАНИЯ
}

(C) Машанов А. А.

кандидат технических наук, доцент

кафедра общей физики

Бурятский государственный университет

670000, Улан-Удэ, ул. Смолина, 24а

E-mail: mashanov@bsu.ru

(C) Мантатов В. В.

доктор физико-математических наук, доцент

кафедра общей физики

Бурятский государственный университет

670000, Улан-Удэ, ул. Смолина, 24а

E-mail: manv999@bsu.ru

(C) Сандитов Д. С.

доктор физико-математических наук, профессор

кафедра общей физики

Бурятский государственный университет

670000, Улан-Удэ, ул. Смолина, 24а

E-mail: sanditov@bsu.ru

Предлагается расчет температурной зависимости энергии активации процесса стеклования аморфных органических полимеров и неорганических стекол с привлечением уравнения Вильямса-Ландела-Ферри для времени релаксации (вязкости). Проведенные оценки находятся в удовлетворительном согласии с экспериментом. Обсуждается природа зависимости процесса стеклования. В рамках модели делокализованных атомов резкий рост энергии активации в области стеклования объясняется возрастанием потенциала конфигурационного изменения структуры при охлаждении стеклообразующего расплава.

Ключевые слова: стеклование, вязкость, время релаксации, энергия активации, аморфные органические полимеры, делокализация атомов, стеклообразующий расплав

\section{Для цитирования:}

Машанов А. А., Мантатов В. В., Сандитов Д. С. О температурной зависимости энергии активации процесса стеклования// Вестник Бурятского государственного университета. Химия. Физика. 2019. Вып 4. С. 47-54.

Природа перехода аморфного вещества из жидкого (высокоэластического) в стеклообразное состояние остается одной из нерешенных проблем. Стеклование жидкости тесно связано с повышением вязкости при охлаждении расплава. При этом энергия активации процесса стеклования, как правило, совпадает с энергией активации вязкого течения (времени релаксации) в области перехода жидкостьстекло. 
Температурная зависимость вязкости простых маловязких жидкостей типа ацетона успешно описывается уравнением Я.И. Френкеля [1]

$$
\eta=\eta_{0} \exp \left(\frac{U}{R T}\right)
$$

с постоянной энергией активации $\mathrm{U}=$ const. Здесь $\eta_{0}-$ предэкспоненциальный множитель, $R$ - газовая постоянная. Однако для стеклообразующих расплавов в формуле (1) энергия активации в области стеклования довольно сильно зависит от температуры: $\mathrm{U}=\mathrm{U}(\mathrm{T})$. В связи с этим предложены эмпирические уравнения вязкости, которые в неявном виде учитывают эту зависимость U(T). Среди них широкое распространение получило соотношение ВЛФ (Вильямса-ЛанделаФерри) [2, 3]

$$
\ln a_{T}=-C_{1} \frac{T-T_{g}}{T-T_{g}+C_{2}}, \quad \quad a_{T}=\frac{\tau(T)}{\tau\left(T_{g}\right)} \cong \frac{\eta(T)}{\eta\left(T_{g}\right)},
$$

где $C_{1}$ и $C_{2}$ - эмпирические постоянные, $T_{g}$ - температура стеклования, $\tau-$ время релаксации. Оправданность этого уравнения показана во многих работах для различных стеклующихся систем [2-4].

Ранее был предложен вывод этого выражения без конкретизации функций $\tau(T)$ и $\eta(T)[5]$

$$
\begin{aligned}
\ln a_{T} & =-\left(\frac{A^{2}}{B}\right) \frac{T-T_{g}}{T-T_{g}+\left(\frac{A}{B} T_{g}\right)}, \\
A & =-\left.\frac{\partial \ln \eta}{\partial\left(T / T_{g}\right)}\right|_{T=T_{g}}, \\
B & =\left.\frac{1}{2} \frac{\partial^{2} \ln \eta}{\partial\left(T / T_{g}\right)^{2}}\right|_{T=T_{g}},
\end{aligned}
$$

Было использовано разложение в ряд функции $\ln \eta(T)$ вблизи $T_{g}$ по малому безразмерному параметру $\lambda=\left(T-T_{g}\right) / T_{g}[5]$.

Таким образом, постоянные уравнения ВЛФ (2) приобретают физический смысл, выражаясь через производные $A$ и $B$

$$
\begin{aligned}
& C_{1}=\frac{A^{2}}{B}, \\
& C_{2}=\frac{A}{B} T_{g} .
\end{aligned}
$$

Настоящая работа посвящена оценке температурной зависимости энергии активации процесса стеклования $U(T)$ с привлечением данных о параметрах уравнения ВЛФ $C_{1}$ и $C_{2}$. 
А. А. Машанов, В. В. Мантатов, Д. С. Сандитов. О температурной зависимости энергии активации процесса стеклования

\section{Расчет температурной зависимости энергии активации стеклования с помощью уравнения Вильямса-Ландела-Ферри}

Легко убедиться в том, что уравнение ВЛФ (2) алгебраически выводится из известного соотношения Фогеля-Фульчера-Таммана [4]

$$
\eta=\eta_{0} \exp \left(\frac{B_{\mathrm{o}}}{T-T_{\mathrm{o}}}\right)
$$

где эмпирическая постоянная $B_{0}$ имеет размерность температуры и измеряется в кельвинах. При этом их параметры оказываются связанными равенствами

$$
\begin{gathered}
B_{\mathrm{o}}=C_{1} C_{2}, \\
T_{\mathrm{o}}=T_{g}-C_{2} .
\end{gathered}
$$

Из сравнения формул для вязкости (1) и (8), полагая в них предэкспоненциальные множители равными, получаем следующую зависимость энергии активации вязкого течения от температуры

$$
U=\frac{B_{\mathrm{o}} R T}{T-T_{\mathrm{o}}}
$$

Поскольку для многих аморфных полимеров и неорганических стекол известны значения параметров уравнения ВЛФ $C_{1}$ и $C_{2}$ [2-4], с помощью выражений (9) и (10) целесообразно перейти от зависимости (11) к величине $U(T)$ как функции этих параметров

$$
U=\frac{C_{1} C_{2} R T}{T-T_{g}+C_{2}} .
$$

Из данного равенства вытекает простое соотношение для расчета энергии активации процесса стеклования $U_{g}=U\left(T_{g}\right)$ при температуре стеклования $T=T_{g}$

$$
U_{g}=C_{1} R T_{g} .
$$

Для листового (оконного) силикатного стекла, например, $C_{1}$ и $T_{\mathrm{g}}$ равны, соответственно [6]: $C_{1}=36$ и $T_{\mathrm{g}}=807 \mathrm{~K}$, откуда имеем $\left(\mathrm{R}=8.3\right.$ Дж/(моль.К)): $\mathrm{U}_{\mathrm{g}}=241$ кДж/моль. Методы релаксационной спектрометрии $[4,6]$ для этого стекла приводят практически к такому же значению $\mathrm{U}_{\mathrm{g}}=242$ кДж/моль. Для полиизобутилена $\left(T_{g}=202 \mathrm{~K}, C_{1}=38\right)$ и натурального каучука $\left(T_{g}=300 \mathrm{~K}, C_{1}=38\right)$ значения $U_{g}$, равные, соответственно, 64 кДж/моль и 95 кДж/моль, также согласуются с данными, полученными методами релаксационной спектрометрии [6].

Известно, что многие стеклующиеся системы переходят из жидкого в твердое стеклообразное состояние практически при одной и той же вязкости $\eta_{\mathrm{g}} \approx \mathrm{const} \approx$ $10^{12}$ Па·с (приближенное правило постоянства вязкости при $T_{g}$ ). Высокотемпературный предел вязкости $\eta_{0} \approx \eta(\mathrm{T} \rightarrow \infty)$ также оказывается в первом приближении постоянным $\eta_{0} \approx$ const $\approx 10^{-3,5}$ Па·c. Поэтому энергию активации при температуре стеклования $U_{g}=U\left(T_{g}\right)$ можно оценить по формуле (1) при $T=T_{g}, \eta_{\mathrm{g}} \approx \eta\left(T_{g}\right) \approx 10^{12}$ Па с и и $\eta_{0} \approx 10^{-3,5}$ Па $\cdot$ с [4]

$$
U_{g}=\left(\ln \frac{\eta_{g}}{\eta_{o}}\right) R T_{g} \approx 36 R T_{g},
$$

что практически совпадает с соотношением (13). 
Следовательно, слабая зависимость параметра уравнения ВЛФ $C_{1}$ от природы стекол: $\mathrm{C}_{1} \approx$ const $\approx 35-40$ (табл. 1) [2, 4] объясняется приближенным постоянством вязкостей $\eta_{\mathrm{g}}$ и $\eta_{0}$ :

$$
C_{1}=\ln \left(\frac{\eta_{g}}{\eta_{0}}\right) .
$$

Таким образом, для расчета $U(T)$ в области стеклования по формуле (12) необходимо знать значения трех величин: $C_{1}, C_{2}$ и $T_{g}$. Данные о параметрах уравнения ВЛФ $C_{1}$ и $C_{2}$, а также о температуре стеклования $T_{g}$, для многих аморфных органических полимеров имеются в книге Ферри [2], а для неорганических стекол - в монографии Сандитова и Бартенева [4]. Один из способов определения $C_{1}$ и $C_{2}$ описан в этой книге $[4$, c. 86$]$.

Таблица

Параметры уравнения ВЛФ (2) и энергия активации процесса стеклования при $T=T_{g}$ для аморфных органических полимеров и неорганических стекол (использованы данные [2, 4])

\begin{tabular}{|c|c|c|c|c|c|}
\hline \multirow{2}{*}{ № } & \multirow{2}{*}{$\begin{array}{l}\text { Аморфные } \\
\text { вещества }\end{array}$} & \multirow{2}{*}{$\begin{array}{c}T_{g} \\
\mathrm{~K}\end{array}$} & \multirow{2}{*}{$C_{1}$} & \multirow{2}{*}{$\begin{array}{c}C_{2}, \\
\mathrm{~K}\end{array}$} & \multirow{2}{*}{$\begin{array}{c}\mathrm{U}_{\mathrm{g}} \\
\text { кДж/моль }\end{array}$} \\
\hline & & & & & \\
\hline \multirow[t]{2}{*}{1.} & $\mathrm{PbO}-\mathrm{SiO}_{2}$ & & & & \\
\hline & PbO, мол. \% 42.07 & 711 & 36 & 250 & 212 \\
\hline \multirow[t]{2}{*}{2.} & $\mathrm{Na}_{2} \mathrm{O}-\mathrm{B}_{2} \mathrm{O}_{3}$ & & & & \\
\hline & $\mathrm{Na}_{2} \mathrm{O}$, мол. \% 2.8 & 544 & 29 & 130 & 131 \\
\hline 3. & Листовое стекло & 807 & 36 & 305 & 241 \\
\hline 4. & Полиизобутилен & 202 & 38 & 104 & 64 \\
\hline 5. & Поливинилацетат & 305 & 38 & 104 & 96 \\
\hline 6. & Полиуретан & 238 & 36 & 33 & 71 \\
\hline 7. & Натуральный каучук & 300 & 38 & 54 & 95 \\
\hline
\end{tabular}

$\mathrm{U}_{\mathrm{g}}$ - расчет по формуле (13).

На рис. 1 приводится температурная зависимость энергии активации процесса стеклования для листового силикатного стекла. Кривая — расчет по формуле (12), а точки - экспериментальные данные. Экспериментальные точки получены из данных о вязкости $\lg \eta(T)$ с помощью уравнения (1), из которого следует

$$
U=2.3 R T\left[\lg \eta(T)-\lg \eta_{0}\right]
$$

Как видно, точки ложатся на расчетную кривую, что фактически подтверждает хорошую применимость формулы ВЛФ. Аналогичные графики $U-T$ построены нами для ряда органических аморфных полимеров (см., например, для поливинилацетата рис. 2). 
А. А. Машанов, В. В. Мантатов, Д. С. Сандитов. О температурной зависимости энергии активации процесса стеклования

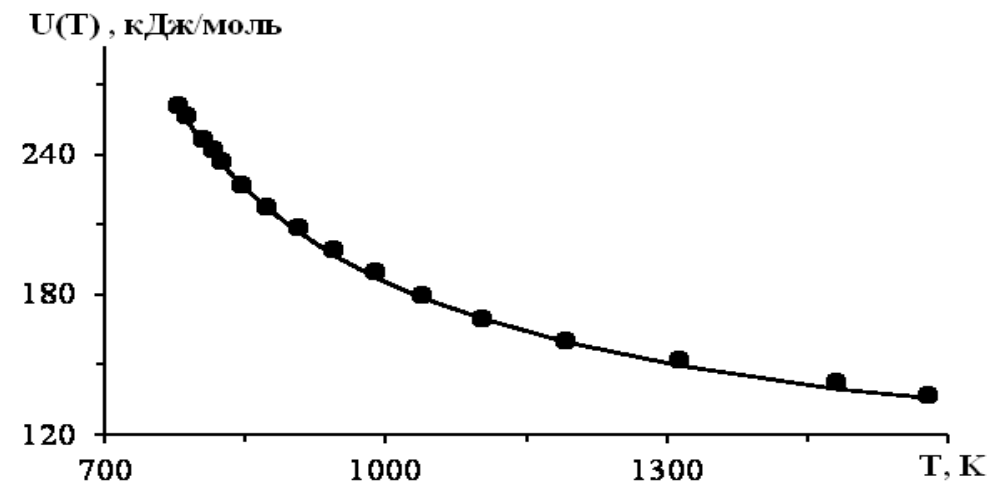

Рис. 1. Температурная зависимость энергии активации стеклования U(T-Tg) для листового силикатного стекла. Точки - экспериментальные данные, кривая расчет по формуле (12).

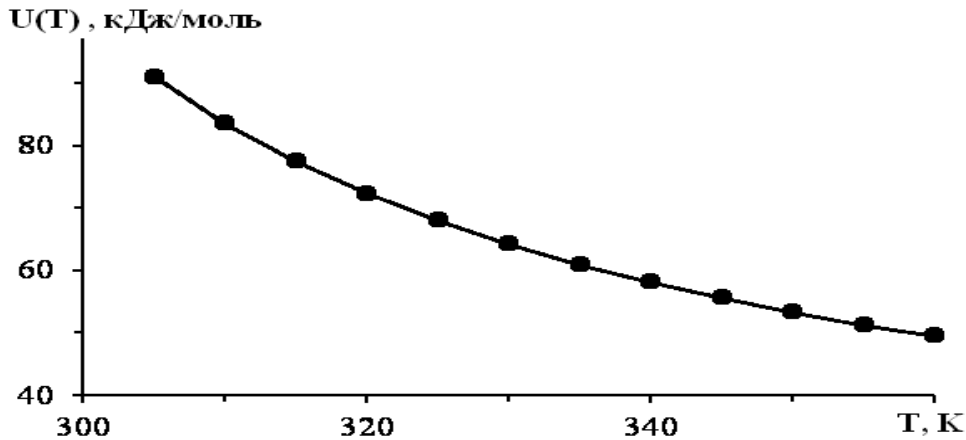

Рис. 2. Температурная зависимость энергии активации стеклования U(T-Tg) для поливинилацетата. Точки - экспериментальные данные, кривая расчет по уравнению (12).

В табл. 1 для некоторых стекол и полимеров приводятся значения параметров $\left(C_{1}, C_{2}\right.$ и $\left.T_{g}\right)$, входящих в соотношение $(12)$, а также величина $U_{g}$, рассчитанная по формуле (13).

\section{О природе зависимости $U(T)$}

Основные закономерности процесса стеклования в качественном отношении оказываются одинаковыми для подавляющего большинства аморфных веществ независимо от их природы: для неорганических стекол, аморфных органических полимеров, металлических аморфных сплавов, водных растворов, халькогенидов, что подтверждается наличием в области стеклования универсальных уравнений и правил [4-7].

Исходим из представления о том, что физической причиной резкого повышения энергии активации процесса стеклования с понижением температуры является конфигурационное изменение структуры стеклообразующего расплава. 
В рамках модели делокализованных атомов $[8,9]$ у стеклообразующих жидкостей энергия активации процесса стеклования (вязкого течения) в уравнении (1) представляет собой сумму двух слагаемых

$$
U=U_{\infty}+U_{s}(T),
$$

где $U_{\infty}$ - потенциал перехода кинетической единицы (атома, молекулы) в новое локальное положение, $U_{S}(T)$ - потенциал конфигурационного изменения структуры, который является функцией температуры,

$$
U_{s}(T)=R T\left[\exp \left(\frac{\Delta \varepsilon_{e}}{R T}\right)-1\right] .
$$

Здесь $\Delta \varepsilon_{e}$ - энергия делокализации атома (его предельного смещения из локального равновесного положения). Делокализация атома обусловлена перегруппировкой соседних частиц (флуктуацией ближнего порядка) и отражает локальное конфигурационное изменение структуры.

При повышенных температурах $R T>>\Delta \varepsilon_{e}$ потенциал конфигурационного структурного изменения равен нулю $U_{S}(T)=0$ и уравнение вязкости (1) с учетом (21) и (22) переходит в обычную аррениусовскую (френкелевскую) зависимость с постоянной энергией активации $U=U_{\infty}$

$$
\eta=\eta_{0} \exp \left(\frac{U_{\infty}}{R T}\right) .
$$

С этой точки зрения величина $U_{\infty}$ оказывается высокотемпературным пределом энергии активации вязкого течения.

При низких температурах, в области стеклования, энергия делокализации атома $\Delta \varepsilon_{e}$ становится сравнимой с энергией тепловых колебаний решетки $(\sim 3 R T)$ и относительное число делокализованных атомов $\left(N_{e} / N\right)$, ответственных за текучесть выше $T_{g}$, существенно уменьшается по закону $\exp \left(-\Delta \varepsilon_{e} / k T\right)$ [9]. Структура расплава уплотняется и для активационного перескока атома в новое положение требуется предварительное локальное конфигурационное изменение («разрыхление») структуры вблизи него: потенциал конфигурационного структурного изменения $U_{S}(T)$ резко возрастает. Этим объясняется практически экспоненциальный рост энергии активации текучести в области стеклования.

Идея разделения энергии активации текучести на две составляющие была высказана Я. И. Френкелем [1]. Дальнейшее развитие она получила в работах Филиповича [10] и Немилова [11]. При более строгом подходе под $U(T)$ следует понимать свободную энергию активации, как это принято в классических работах Эйринга [12].

Таким образом, конфигурационное изменение структуры, которое описывается в рамках модели делокализованных атомов, оказывается ответственным за температурную зависимость энергии активации процесса стеклования (вязкого 
А. А. Машанов, В. В. Мантатов, Д. С. Сандитов. О температурной зависимости энергии активации процесса стеклования

течения) в области перехода из жидкого (высокоэластического) состояния в стеклообразное.

\section{Заключение}

Для аморфных органических полимеров и неорганических стекол рассмотрены способы расчета температурной зависимости энергии активации процесса стеклования $U(T)$. Данная зависимость $U(T)$ удовлетворительно описывается вблизи $T_{g}$ с привлечением известного уравнения Вильямса-Ландела-Ферри для вязкости (времени релаксации). В рамках модели делокализованных атомов резкий рост энергии активации в области стеклования объясняется возрастанием потенциала конфигурационного изменения структуры при охлаждении стеклообразующего расплава.

Работа выполнена при финансовой поддержке Министерства науки и высшего образования РФ (грант № 3.5406.2017/8.9).

\section{Литература}

1. Френкель Я. И. Кинетическая теория жидкостей. - М.-Л.: Изд-во АН СССР, 1945. - 279 c

2. Ферри Дж. Вязкоупругие свойства полимеров. - Изд-во ИЛ, 1963. - 535 с.

3. Williams M. L., Landel R. F., Ferry J. D. The temperature dependence of relaxation mechanisms in amorphous polymers and other glass-forming liquids // J. Amer. Chem. Soc. 1955. - V. 77, № 14. - P. 3701-3707.

4. Сандитов Д. С., Бартенов Г. М. Физические свойства неупорядоченных структур. - Новосибирск: Наука, 1982. - 261 с.

5. Сандитов Д. С., Разумовская И. В. Новый подход к обоснованию уравнения Вильямса-Ландела-Ферри // Высокомолек. соед. Сер. А. - 2018. — Т. 60, № 2. - С. 110115.

6. Бартенев Г. М., Сандитов Д. С. Релаксационные процессы в стеклообразных системах. - Новосибирск: Наука, 1986. - 238 с.

7. Ростиашвили В. Г., Иржак В. И., Розенберг Б. А. Стеклование полимеров. Л.: Химия, 1987. - 197 с.

8. Сандитов Д. С. Модель делокализованных атомов в физике стеклообразного состояния // Ж. экспериментальной и теоретической физики. — 2012. — Т. 142, Вып. 1(7). - С. $123-137$.

9. Сандитов Д. С., Ожован М.И. Релаксационные аспекты перехода жидкостьстекло // Успехи физических наук. — 2019. — Т. 189, № 2. — С. 113-133.

10. Филипович В. Н. Вакансионно-диффузионная теория вязкости стекол // Физика и химия стекла. - 1975. - Т. 1, № 3. - С. 256-264.

11. Немилов С. В. Валентно-конфигурационная теория вязкого течения переохлажденных стеклообразующих жидкостей и ее экспериментальное обоснование // Физика и химия стекла. - 1978. - Т. 4, № 2. - С. 129-148.

12. Глесстон С., Лейдлер К., Эйринг Г. Теория абсолютных скоростей реакций. М.: Изд-во ИЛ, 1948. — 583 с. 


\section{ABOUT TEMPERATURE DEPENDENCE OF ENERGY ACTIVATION} OF THE GLASS PROCESS

\section{Mashanov A. A.}

Candidate of Engineering Sciences, associate Professor

Department of General Physics

Buryat State University

670000, Ulan-Ude, Smolina Str., 24a

E-mail: mashanov@bsu.ru.

Mantatov $V . V$.

Doctor of Physical and Mathematical Sciences, associate Professor

Department of General Physics

Buryat State University

670000, Ulan-Ude, Smolina Str., 24a

E-mail: manv999@bsu.ru

Sanditov D. S.

Doctor of Physical and Mathematical Sciences, Professor

Department of General Physics

Buryat State University

670000, Ulan-Ude, Smolina Str., 24a

E-mail: sanditov@bsu.ru

It is proposed to calculate the temperature dependence of the activation energy of the glass transition process of amorphous organic polymers and inorganic glasses using the WilliamsLandel-Ferry equation for the relaxation time (viscosity). The estimates made are in satisfactory agreement with the experiment. The nature of the dependence of the glass transition process is discussed. In the framework of the model of delocalized atoms, a sharp increase in the activation energy in the glass transition region is explained by an increase in the potential for configuration changes in the structure during cooling of the glass-forming melt. Keywords: glass transition, viscosity, relaxation time, activation energy, amorphous organic polymers, delocalization of atoms, glass-forming melt 